\title{
Validation of High-Speed Turbulent Boundary Layer and Shock-Boundary Layer Interaction Computations with the OVERFLOW Code
}

\author{
A. B. Oliver*, R. P. Lillard† G. A. Blaisdell; and A. S. Lyrintzis ${ }^{\ddagger}$ \\ School of Aeronautics and Astronautics \\ Purdue University \\ West Lafayette, IN 47907
}

\begin{abstract}
The capability of the OVERFLOW code to accurately compute high-speed turbulent boundary layers and turbulent shock-boundary layer interactions is being evaluated. Configurations being investigated include a Mach 2.87 flat plate to compare experimental velocity profiles and boundary layer growth, a Mach 6 flat plate to compare experimental surface heat transfer, a direct numerical simulation (DNS) at Mach 2.25 for turbulent quantities, and several Mach 3 compression ramps to compare computations of shock-boundary layer interactions to experimental laser doppler velocimetry (LDV) data and hot-wire data. The present paper describes outlines the study and presents preliminary results for two of the flat plate cases and two small-angle compression corner test cases.
\end{abstract}

\section{Nomenclature}

$C_{f} \quad$ Local skin friction coefficient

$M \quad$ Local Mach number

$V \quad$ Velocity

$\delta \quad$ Boundary layer thickness

$\delta^{*} \quad$ Boundary layer displacement thickness

$\theta \quad$ Boundary layer momentum thickness

$P_{s} \quad$ Static pressure

$T \quad$ Temperature

$x \quad$ Streamwise coordinate

$y \quad$ Crossflow coordinate

$z \quad$ Wall-normal coordinate

$L_{\text {sep }}$ Separation length

Subscripts

Ref Reference value for data reduction

$0 \quad$ Stagnation condition

\section{Introduction}

useful thermal analysis tool for the space shuttle and future space vehicles needs to accurately model - 1 turbulent boundary layers in order to predict the heat transfer on the surface. Shock-boundary layer

*Graduate Research Assistant, Student Member AIAA.

${ }^{\dagger} \mathrm{PhD}$ Candidate, Student Member AIAA; Aerospace Engineer, NASA/JSC, Aerosciences and CFD Branch

$¥$ Associate Professor, Senior Member AIAA.

$\S$ Professor, Associate Fellow AIAA. 
interactions cause a significant increase in wall heating in the reattachment region of a shock-separated boundary layer, so they must be accurately modeled as well. The present work is carried out to continue the development of the capability to model high-speed compressible flows over complex vehicles. The goal of the current project is to evaluate the performance of the turbulence models in the OVERFLOW code ${ }^{1,2}$ for high-speed, non-reacting flow.

The OVERFLOW code is an overset (chimera) grid Navier-Stokes solver, which makes it ideal for computing the flow fields over complex geometries. ${ }^{3}$ The code utilizes the Swanson/Turkel matrix dissipation model $^{4}$ which, with the correct parameters, ${ }^{5}$ allows the central-differenced scheme to mimic a Total Variation Diminishing (TVD) upwind biased scheme in regions of flow discontinuities. This method takes advantage of the speed of central differencing while maintaining the accuracy of the upwind TVD scheme. The idea of using OVERFLOW as a thermal analysis tool has received some criticism based on the fact that it was designed to compute aerodynamic forces in transonic and low supersonic flows. Previous work ${ }^{5,6}$ has indicated that it is indeed capable of accurately modeling laminar hypersonic flows and does an adequate job of capturing heat transfer at those speeds. The present study is now directing attention at validating the use of OVERFLOW for high-speed turbulent flows.

Three turbulence models in OVERFLOW will be considered in this paper: the Spalart-Allmaras model, ${ }^{7}$ the SST model, ${ }^{8}$ and the Lag model. ${ }^{9}$ Results from each model will be compared to several different experiments and direct numerical simulation (DNS) computations in order to benchmark their behavior.

\section{A. Code Description}

OVERFLOW is a structured (overset) grid Navier-Stokes flow solver. It uses a finite-difference formulation with flow quantities stored at the grid nodes. OVERFLOW has central- and Roe upwind-difference options, and uses a diagonalized, implicit approximate factorization scheme for time advancement. Local timestepping, multigrid techniques and grid sequencing are all used to accelerate convergence to a steady state solution. In this study, a 2nd-order central differencing with Swanson/Turkel type 2nd/4th-order matrix dissipation is utilized. For a complete discussion of the scheme, see Olsen et al. ${ }^{5}$

\section{B. Turbulence Models}

OVERFLOW has numerous turbulence models coded in, but this study will focus on three models, the Spalart-Allmaras (SA) model, ${ }^{7}$ the SST model, ${ }^{8}$ and the Lag model. ${ }^{9}$ The turbulence models included in the code all make use of the Boussinesq approximation, meaning that the effects of the Reynolds stress terms are included in the Navier-Stokes equations through an eddy viscosity. The SA model is a popular model for aerodynamic flows that solves a single partial differential equation directly for the eddy viscosity. The SST model is a two-equation model which is a hybrid of the $\mathrm{k}-\epsilon$ and $\mathrm{k}-\omega$ turbulence models and solves equations for the turbulent kinetic energy and the specific dissipation rate. The eddy viscosity is then derived from an algebraic relation that chooses the minimum value between the standard formulation based on the turbulence kinetic energy and the specific dissipation rate and a formulation based on an assumed value of the normalized Reynolds shear stress. The Lag model is a recent extension of the SST model that accounts for non-equilibrium effects by carrying an additional differential equation that relaxes the eddy viscosity to the equilibrium value. This third equation accounts for the time required for the turbulence to respond to changes in the mean flowfield. Each of the test cases is computed with all three turbulence models to allow comparisons of the models.

\section{Study Objectives}

The objective of the current study is to benchmark the performance of these turbulence models, as implemented within OVERFLOW, for the high-supersonic to hypersonic regimes experienced by the space shuttle during ascent. Surface properties, such as surface pressure and heat transfer, are the primary variables of interest, however to aid in the development and implementation of improved models and model corrections, profiles of flow variables and turbulent quantities are also being generated.

This paper presents the surface properties and select profiles for a few of the test cases that have been completed. There is much work that remains to be done in this study, however in the interest of generating useful feedback, the entire project will be outlined. 


\section{Test Cases}

The test cases are each chosen to address a primary objective in this study. In order to validate the code's ability to capture a zero pressure gradient boundary layer, several flat plate cases will be run. Twodimensional compression ramp experiments have been selected to analyze the shock/boundary layer interaction behavior, namely the size of the separation region, the profiles of turbulent quantities downstream of the shock, and the quality of the prediction in the recovering boundary layer. Since experiments often have to make some compromises in terms of what measurements can be made, multiple experiments have been chosen for both geometries to capture specific features of the turbulent boundary layers. The experiments have been chosen based on perceived quality of the data and Mach number of interest.

\section{A. Flat Plate}

In order to adequately address each relevant feature of a high-speed boundary layer, four datasets have been selected for comparison. An experimental study by Cary ${ }^{10}$ at Mach 6 has been chosen for its surface heating data. A DNS simulation by Gatski ${ }^{11}$ provides two detailed profiles of mean and turbulent properties. Finally, an experiment conducted by Smits, et al. ${ }^{12}$ at Mach 2.87 has profiles at multiple locations upstream of an $8^{\circ}$ compression corner, and will be used for comparison of high Reynolds number experimental velocity profiles and boundary layer growth measurements.

\section{B. Compression Ramp}

Two supersonic compression ramp datasets have been chosen for comparison. The Mach 2.9 experiments of Kuntz ${ }^{13,14}$ will provide two component laser doppler velocimetry (LDV) measurements of turbulent boundary layers on ramps of $8,12,16,20$, and 24 degrees, and the Mach 2.9 series of experiments ${ }^{12,15}$ conducted at the Princeton Gas Dynamics Laboratory will be used to compare turbulence quantities downstream of the shock and for skin friction and surface pressure distributions on ramps of $8,16,20$, and 24 degrees. Settles and Dodson reported in their 1994 shock-boundary layer interaction database ${ }^{16}$ that these two datasets disagree on the magnitude of the Reynolds stresses by as much as a factor of 4 . To date, the authors are not aware of an explanation of this discrepancy, so following the suggestion of Settles and Dodson, these values are taken to be the bounds of the actual Reynolds stresses.

The Mach 3.0 LES simulations of Rizzetta et al. ${ }^{17,18}$ are also being considered for comparison, however difficulties have been encountered while trying to compare to this dataset and it is unknown if these issues will be worked out.

\section{Solution Methods}

\section{A. Grid Generation and Boundary Conditions}

The flat plate test cases use simple single-zone grids that do not model the radius of the plate leading edge, and the viscous wall extends all the way to the inflow plane. The compression ramp cases use a system similar to that depicted in Figure 1. The inflow turbulent velocity profiles given in each experimental case is generated on the long flat plate region upstream of the corner, which is computed together with the ramp as one computation. The OVERFLOW cases are run fully turbulent, so the length of this plate must changed to specify the 'transition point'. The length of the flat plate is chosen such that the momentum thickness in matched to the experiment as closely as possible. The corner and ramp regions are defined by three grids: two high resolution near-wall grids (split for load-balancing on multiple processors) and a grid in the freestream. It was found that the hyperbolic tangent stretching function used for the wall-normal direction did not place the desired number of grid points in the freestream above the shock wave, so the freestream was given its own grid. The final grid is a low resolution sponge grid to contain the leading edge shock wave and prevent it from reflecting back and interfering with the boundary layer development.

The boundary conditions for both grid systems are freestream at the inflow boundary, characteristic freestream on the boundary opposite the viscous wall, and extrapolation at the outflow. The viscous wall is either adiabatic or isothermal, based on the specific dataset being compared to.

It was discovered that adding the flat-top to the compression ramp downstream of the experimental data changed the size of the separation bubble for a large ramp angle. This was taken to mean that disturbances 
from the $0^{\text {th }}$ order extrapolation outflow boundary condition were propagating upstream through the subsonic portion of the boundary layer. While it has not yet been determined if this holds true for smaller ramp angles, the flat tops were also added to these cases for the sake of consistency.
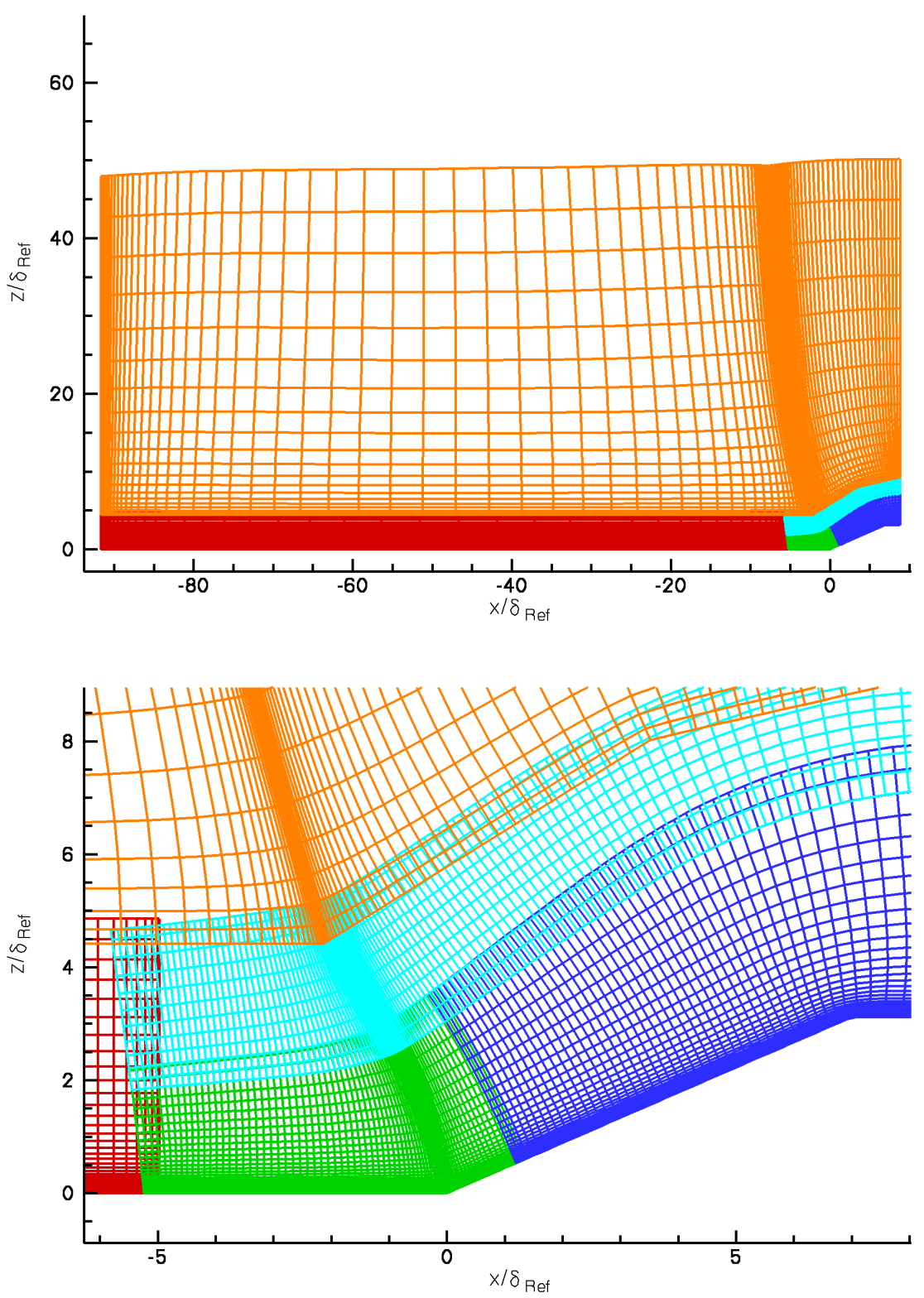

Figure 1. Grid topology for $24^{\circ}$ ramp with every other grid line shown. (a) Overall grid system, (b) Detail view of corner region.

\section{B. Solution Method}

Each of the solutions is initialized to freestream conditions and iterated on a sequence of progressively finer grids until the finest grid level is obtained. Coarse grids are created internally in OVERFLOW by specifying a number of grid levels (three grid levels would be the original fine grid, a medium grid with every other grid point used, and a coarse grid with every fourth grid point used). For this study, three grid levels were used and between 500 and 1,000 iterations were performed on the coarse and medium grid levels to startup the solution. 
In order to rapidly obtain a high quality steady-state solution, the dissipation parameters for the matrix dissipation scheme ${ }^{4}$ are ramped down to their optimum level as the solution converges. The four parameters controlled are the 2nd and 4th-order dissipation coefficients, $\kappa_{2}$ and $\kappa_{4}$, and the eigenvalue limiters $\mathrm{V}_{\epsilon_{\eta}}$ and $\mathrm{V}_{\epsilon_{\ell}}$ (DIS2, DIS4, VEPSN, and VEPSL in OVERFLOW, respectively). The presented solutions were started with these values set to $(10,0.2,1.0,1.0)$ and were ramped down to $(2,0.1,0.3,0.3)$. For more information on this process, see Lillard et al. ${ }^{6}$

The solutions are considered to be converged with the optimal dissipation settings when: (1) the residuals have dropped by more than three orders of magnitude and, (2) the skin friction at several representative locations has converged to a constant value. Grid convergence was determined using the Richardson extrapolation similar to the method of Roache. ${ }^{19}$ The solutions in the present paper were considered to be adequately grid resolved when the average difference between the high resolution skin friction and the extrapolated skin friction in the corner/ramp region was less than $3 \%$. Grid convergence results are presented in Table 1 and Table 3.

Table 1. Inflow matching and grid convergence results for compression ramp test cases

\begin{tabular}{|l|ccccc|}
\hline Case & $\frac{\theta}{\theta_{\text {Target }}}$ & $\frac{\delta^{*}}{\delta_{\text {Target }}^{*}}$ & RMS $\Delta C_{f}$ & Max $\Delta C_{f}$ & $\Delta L_{\text {Sep }}$ \\
\hline $8^{\circ}$ Ramp (SST) & 1.001 & 1.002 & $0.78 \%$ & $2.0 \%$ & $2.1 \%$ \\
$8^{\circ}$ Ramp (SA) & 0.986 & 0.993 & $1.8 \%$ & $8.5 \%$ & $7.9 \%$ \\
$8^{\circ}$ Ramp (Lag) & 0.994 & 0.996 & $1.76 \%$ & $7.4 \%$ & $3.0 \%$ \\
$16^{\circ}$ Ramp (SST) & 0.999 & 1.060 & $2.2 \%$ & $17 \%$ & $2.6 \%$ \\
$16^{\circ}$ Ramp (SA) & 0.995 & 1.063 & $2.1 \%$ & $8.8 \%$ & $0.58 \%$ \\
$16^{\circ}$ Ramp (Lag) & 1.015 & 1.078 & $2.9 \%$ & $14.4 \%$ & $0.77 \%$ \\
\hline
\end{tabular}

\section{Preliminary Results}

Work on this project is continuing; however, several computations have been completed and are presented below. These solutions include the Gatski flat plate and the mean flow portion of the $8^{\circ}$ and $16^{\circ}$ compression corners from the Princeton series of experiments. The reference values in Table 2 are used to scale the output of the following data.

Table 2. Reference values used in data reduction

\begin{tabular}{|c|ccc|}
\hline Case & Flat Plate & $8^{\circ}$ Ramp & $16^{\circ}$ Ramp \\
\hline$M_{\text {Ref }}$ & 2.25 & 2.87 & 2.85 \\
$V_{\text {Ref }}(\mathrm{m} / \mathrm{s})$ & 260.9 & 590 & 580 \\
$\delta_{\text {Ref }}(\mathrm{mm})$ & 3.8 & 26 & 26 \\
$P_{\text {Ref }}(\mathrm{kPa})$ & - & 22.52 & 23.56 \\
\hline
\end{tabular}

\section{A. Flat Plate}

Two of the flat plate test cases have been run to date, namely the Gatski DNS test case and the feeder plate of the $8^{\circ}$ compression ramp experiment from the Princeton series. The data available from the Gatski dataset includes skin friction and velocity profiles at two points on the flat plate. Table 3 shows the skin friction results for each turbulence model against the DNS value, and Figure 2 shows the two velocity profiles.

The analysis of the flat plate boundary layer upstream of the $8^{\circ}$ compression ramp is given in Table tab:ccfp. The boundary layer displacement thickness and momentum thickness comparisons seem to be within the experimental scatter for all turbulence models (keeping in mind that $\theta$ at $x=-25.4 \mathrm{~mm}$ was used to define the length of the flat plate). The skin friction predictions range from $9 \%$ to $13 \%$ too large for the OVERFLOW solutions. It is difficult to call one turbulence model better than the others based on this data alone. 
Table 3. Gatski flat plate grid convergence and skin friction results

\begin{tabular}{|c|cc|cc|}
\hline Case & RMS $\Delta C_{f}$ & Max $\Delta C_{f}$ & $C_{f} x=7.8 i n$ & $C_{f} x=8.8 i n$ \\
\hline DNS & - & - & $2.59 \times 10^{-3}$ & $2.25 \times 10^{-3}$ \\
SST & $0.53 \%$ & $0.55 \%$ & $2.01 \times 10^{-3}(22 \%)$ & $1.97 \times 10^{-3}(12 \%)$ \\
SA & $0.63 \%$ & $0.65 \%$ & $2.07 \times 10^{-3}(20 \%)$ & $2.03 \times 10^{-3}(9.7 \%)$ \\
Lag & $0.60 \%$ & $1.07 \%$ & $2.02 \times 10^{-3}(22 \%)$ & $2.01 \times 10^{-3}(11 \%)$ \\
\hline
\end{tabular}

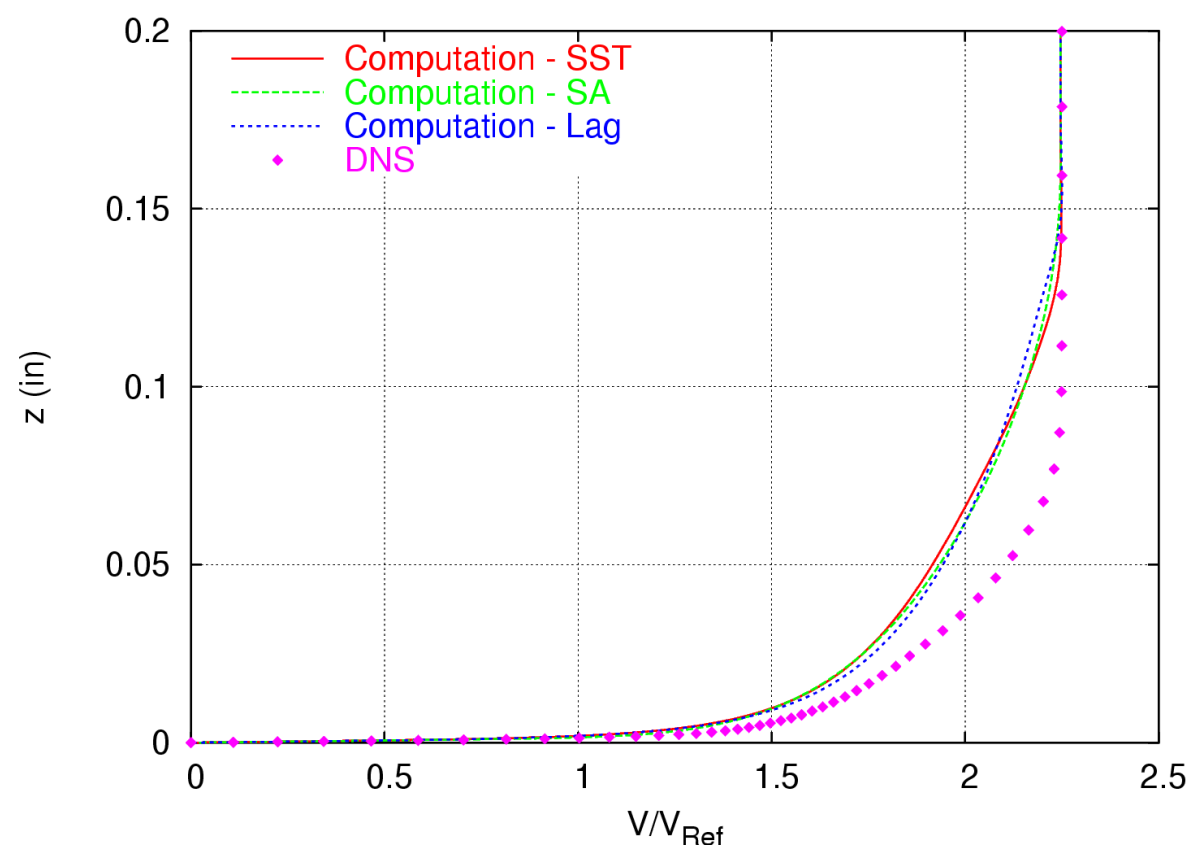

Figure 2. Velocity profile at $x=8.8 \mathrm{in}$ for Gatski flat plate test case.

\section{B. Compression Ramp}

Freestream conditions for the compression ramp cases shown are given in Table 5. Figure 3 shows the skin friction distributions on the $8^{\circ}$ and $16^{\circ}$ ramps. For both of these cases, the skin friction from the upstream boundary layer is too high, and all the OVERFLOW computations indicate separation when it was not observed in the experiments. This aside, the Lag model seems to perform better with increasing shock strength, while the SA performance decreases. The SST model seems prone to separating too early.

Figure 4 shows the static pressure in the flowfield and on the surface of the $8^{\circ}$ ramp. For this case, all of the models do a descent job representing the data. Figure 5, which shows the pressure for the $16^{\circ}$ ramp, is a little more interesting. It appears that, as expected from Figure 3 above, the SST model separates too early and the oversized separation bubble throws off the pressure in the recovering boundary layer. The Lag model predicts the location of the pressure rise very well, but the pressure in the recovering boundary layer falls a little below the measured pressure. The SA model, on the other hand, misses the pressure rise but gets the recovery pressure profile. The reversal in which model better predicted the surface pressure and skin friction seems to indicate that these differences are within the experimental uncertainty.

Looking at the contour plots in Figures 4 and 5, it appears that there are large disturbances and a fat shock in the inviscid flowfield behind the shock wave. This is an artifact of the numerical dissipation scheme used in OVERFLOW, which is known to smear shocks over 5-7 grid points. To see whether or not this was affecting the surface properties, an case was run with a special grid to capture and contain the shock wave. This grid, shown in Figure 6a, is aligned closely with the shock predicted from the $16^{\circ}$ computation. Figure $6 \mathrm{~b}$ shows the skin friction for the $16^{\circ}$ ramp with and without the shock grid. As you can see, the 

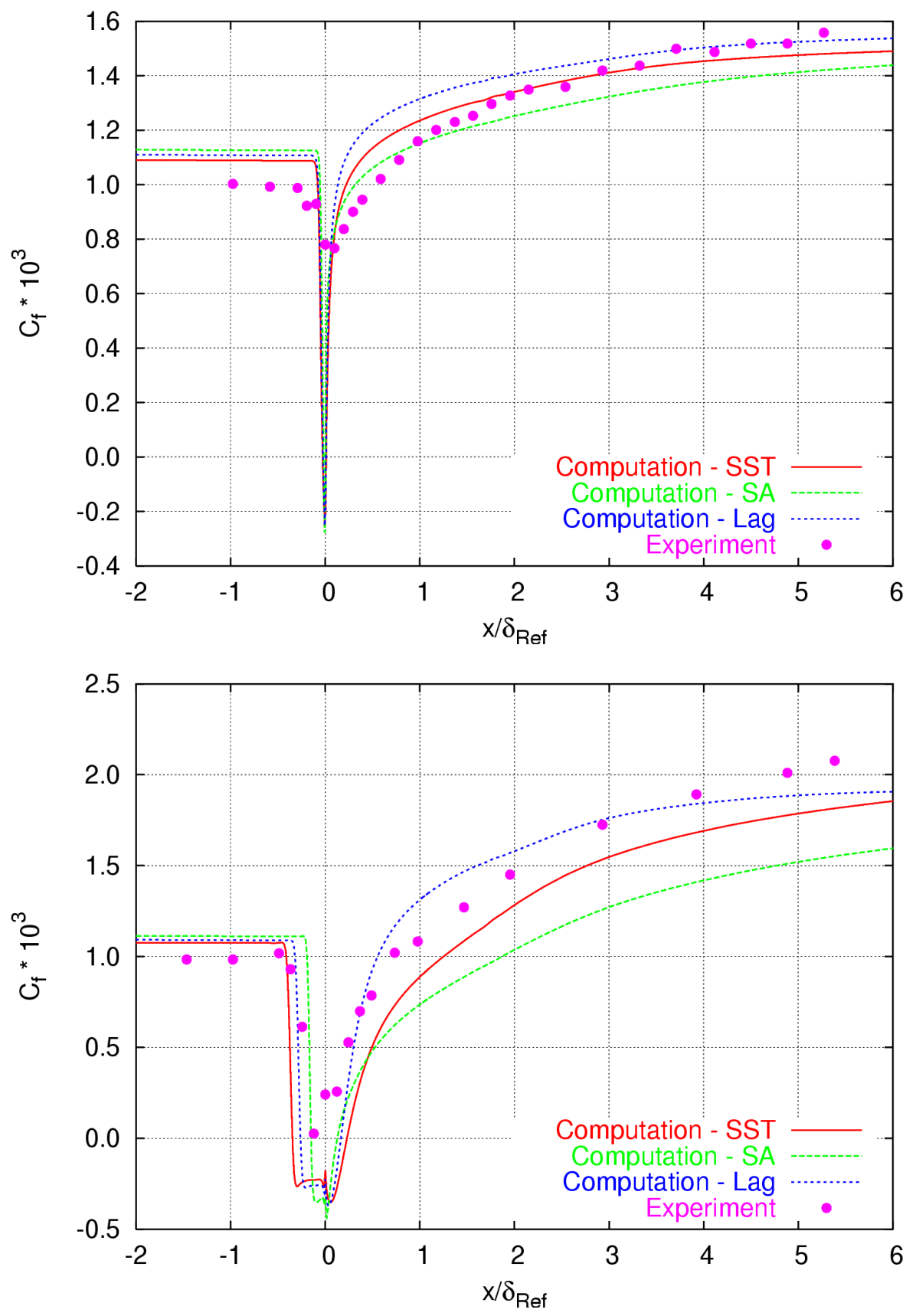

Figure 3. Profiles of skin friction on (a) $8^{\circ} \mathrm{ramp}$, (b) $16^{\circ}$ ramp. 

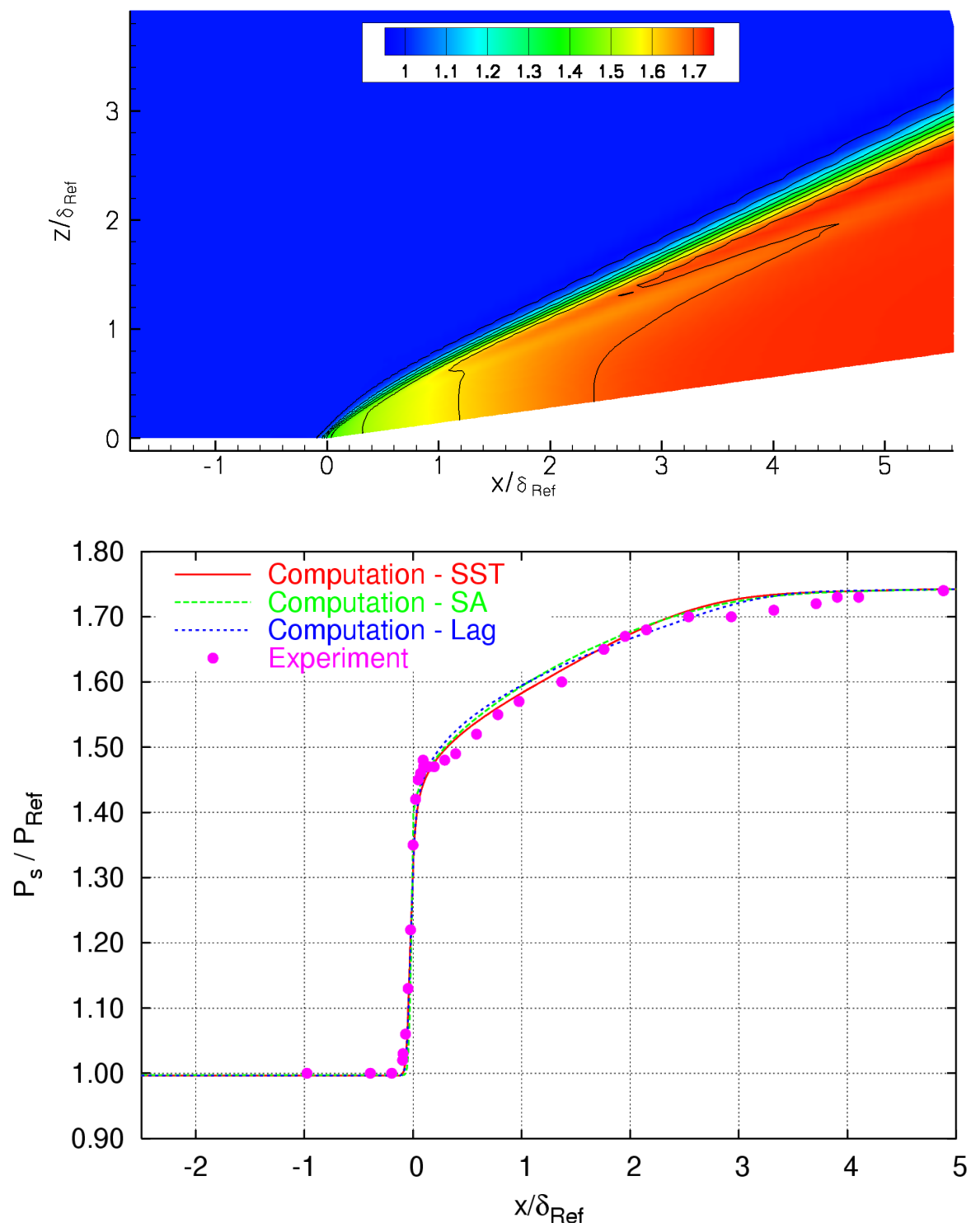

Figure 4. (a) Contours of $\frac{P_{s}}{P_{R e f}}$ and (b) surface pressure on $8^{\circ}$ ramp. 

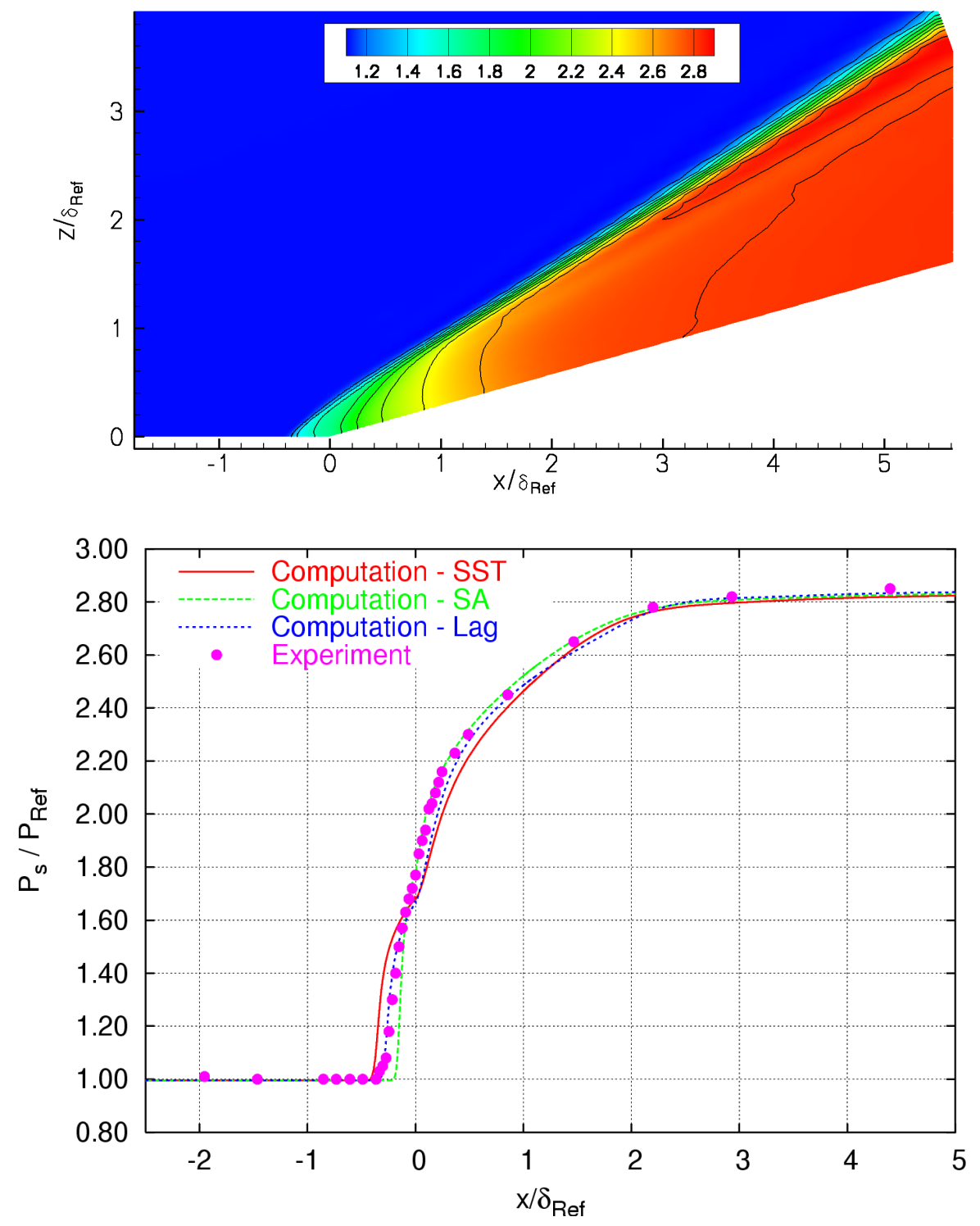

Figure 5. (a) Contours of $\frac{P_{s}}{P_{R e f}}$ and (b) surface pressure on $16^{\circ}$ ramp. 
Table 4. Boundary layer development on feeder flat plate for $8^{\circ}$ compression corner case

\begin{tabular}{|lllll|}
\hline Case & $C_{f}$ & $\delta(\mathrm{mm})$ & $\delta^{*}(\mathrm{~mm})$ & $\theta(\mathrm{mm})$ \\
\hline$x=-50.8 m m$ & & & \\
Experiment & $1.00 \times 10^{3}$ & 26.0 & 6.7 & 1.3 \\
SST & $1.09 \times 10^{3}$ & 24.5 & 6.60 & 1.29 \\
SA & $1.13 \times 10^{3}$ & 27.1 & 6.58 & 1.27 \\
Lag & $1.11 \times 10^{3}$ & 26.1 & 6.61 & 1.28 \\
\hline$x=-25.4 m m$ & & & & \\
Experiment & $1.00 \times 10^{3}$ & 26.5 & 6.6 & 1.3 \\
SST & $1.09 \times 10^{3}$ & 24.8 & 6.71 & 1.30 \\
SA & $1.13 \times 10^{3}$ & 27.4 & 6.66 & 1.28 \\
Lag & $1.11 \times 10^{3}$ & 26.4 & 6.61 & 1.29 \\
\hline$x=-15.2 m m$ & & & & \\
Experiment & $0.990 \times 10^{3}$ & 26.5 & 7.3 & 1.2 \\
SST & $1.09 \times 10^{3}$ & 24.9 & 6.75 & 1.31 \\
SA & $1.13 \times 10^{3}$ & 27.5 & 6.69 & 1.29 \\
Lag & $1.11 \times 10^{3}$ & 26.6 & 6.71 & 1.30 \\
\hline
\end{tabular}

Table 5. Freestream and inflow conditions used in compression ramp computations

\begin{tabular}{|c|ccc|}
\hline Ramp Angle & $8^{\circ}$ & $16^{\circ}$ & $24^{\circ}$ \\
\hline$M_{\infty}$ & 2.87 & 2.85 & 2.84 \\
$T_{o}(\mathrm{k})$ & 280 & 268 & 262 \\
$P_{o}(\mathrm{kPa})$ & 680 & 690 & 690 \\
$\delta(\mathrm{mm})$ & 26 & 26 & 23 \\
$\delta^{*}(\mathrm{~mm})$ & 6.7 & 6.3 & 6.1 \\
$\delta(\mathrm{mm})$ & 1.3 & 1.3 & 1.2 \\
$x_{\text {upstream }}(\mathrm{mm})$ & -25.4 & -25.4 & -63.5 \\
\hline
\end{tabular}

differences are negligible (less than 1\% along the entire length), so the shock grids were left off to ease the load of obtaining grid-converged solutions for the present paper. Shock grids will be used once profiles of mean and turbulent properties are analyzed in detail.

Figures 7 and 8 show velocity and local Mach number profiles for the $8^{\circ}$ and $16^{\circ}$ ramps respectively. For the $8^{\circ}$ ramp, the shock-boundary layer interaction is weak enough that most of the differences between the three turbulence models is small. However, for the $16^{\circ} \mathrm{ramp}$, characteristic features of RANS predictions begin to appear. In each of the profiles downstream of the shock, the flow near the wall slows down too much. The Lag model does a much better job than the other turbulence models in this regard and fits the near-wall data very well for profile 5 , well within the experimental uncertainty.

As the present data shows, these two ramp angles do not really create a strong enough interaction to significantly separate these three turbulence models; solutions for steeper ramp angles will be required. Work is presently underway to obtain steady solutions of the $20^{\circ}$ and $24^{\circ}$ compression corners, however initial predictions have been very poor and often do not converge to a steady solution. One option currently being explored is running OVERFLOW in a time-accurate unsteady calculation and taking time-averages of the surface properties. Figure 9 shows initial results for skin friction and surface pressure the $24^{\circ}$ ramp. The SA model converged to a steady solution, however the correlation with the experimental data is very poor-worse than would have been expected beforehand. The SST solution shown is the time average of an initial unsteady computation. This method looks promising and work is currently underway to make sure that the time resolution is sufficiently fine and the run time is long enough to give accurate statistics. 

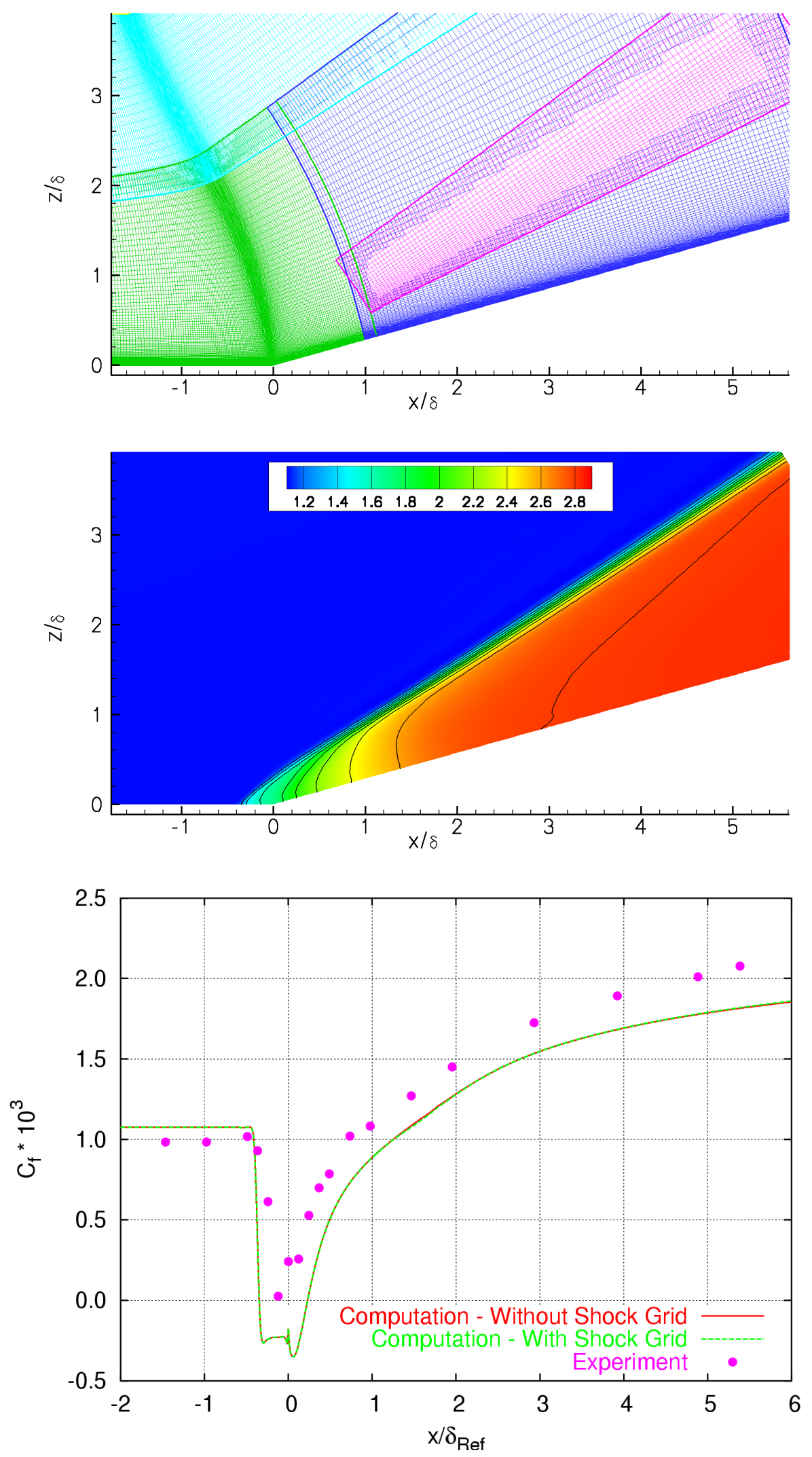

Figure 6. $16^{\circ}$ ramp with shock grid. (a) Grid system, (b) Contours of $\frac{P_{s}}{P_{R e f}}$, (c) Computed skin friction with and without shock grid. 

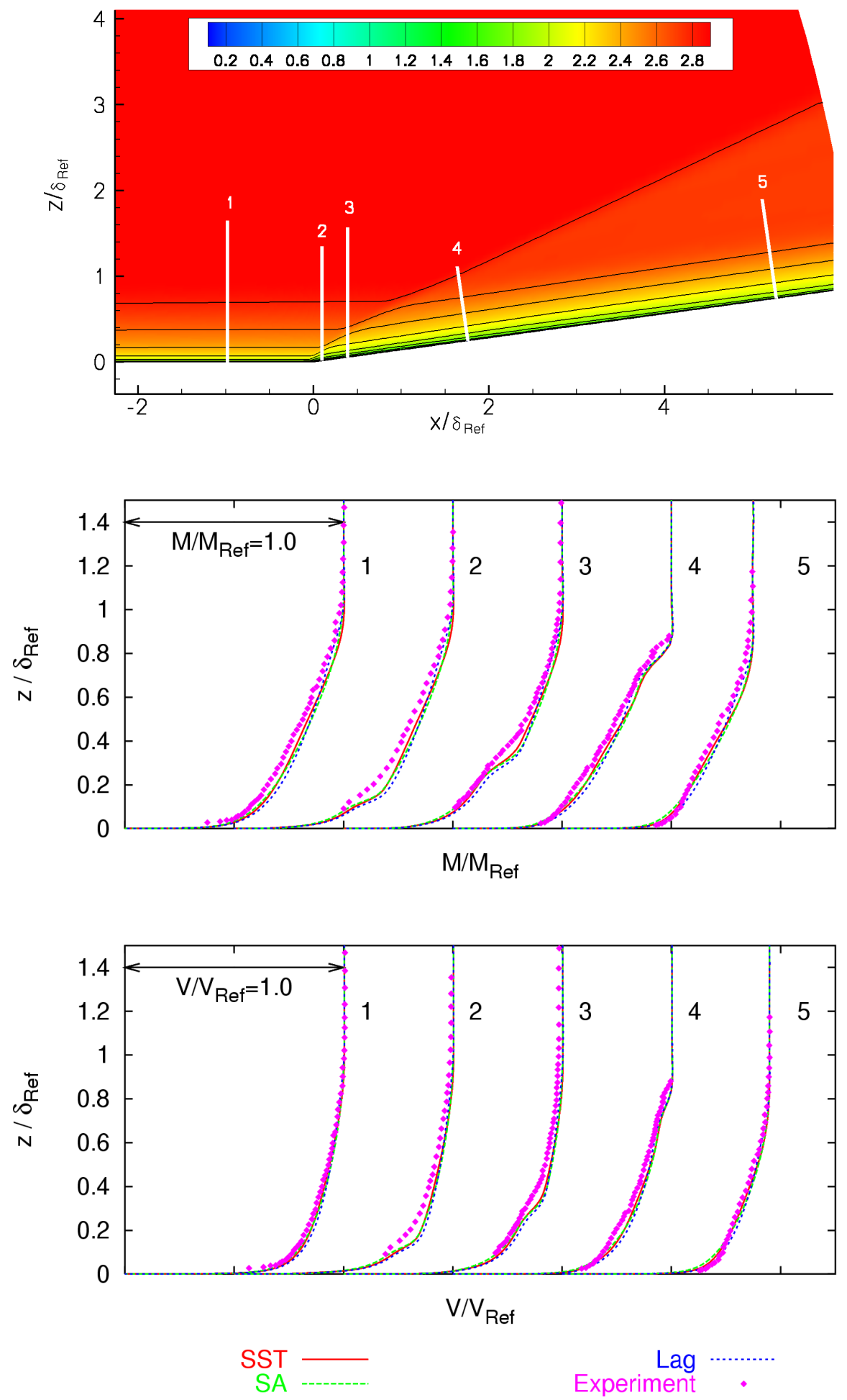

Figure 7. $8^{\circ}$ ramp: (a) Mach contours with profile locations indicated. (b) Mach profiles. (c) Velocity profiles. 

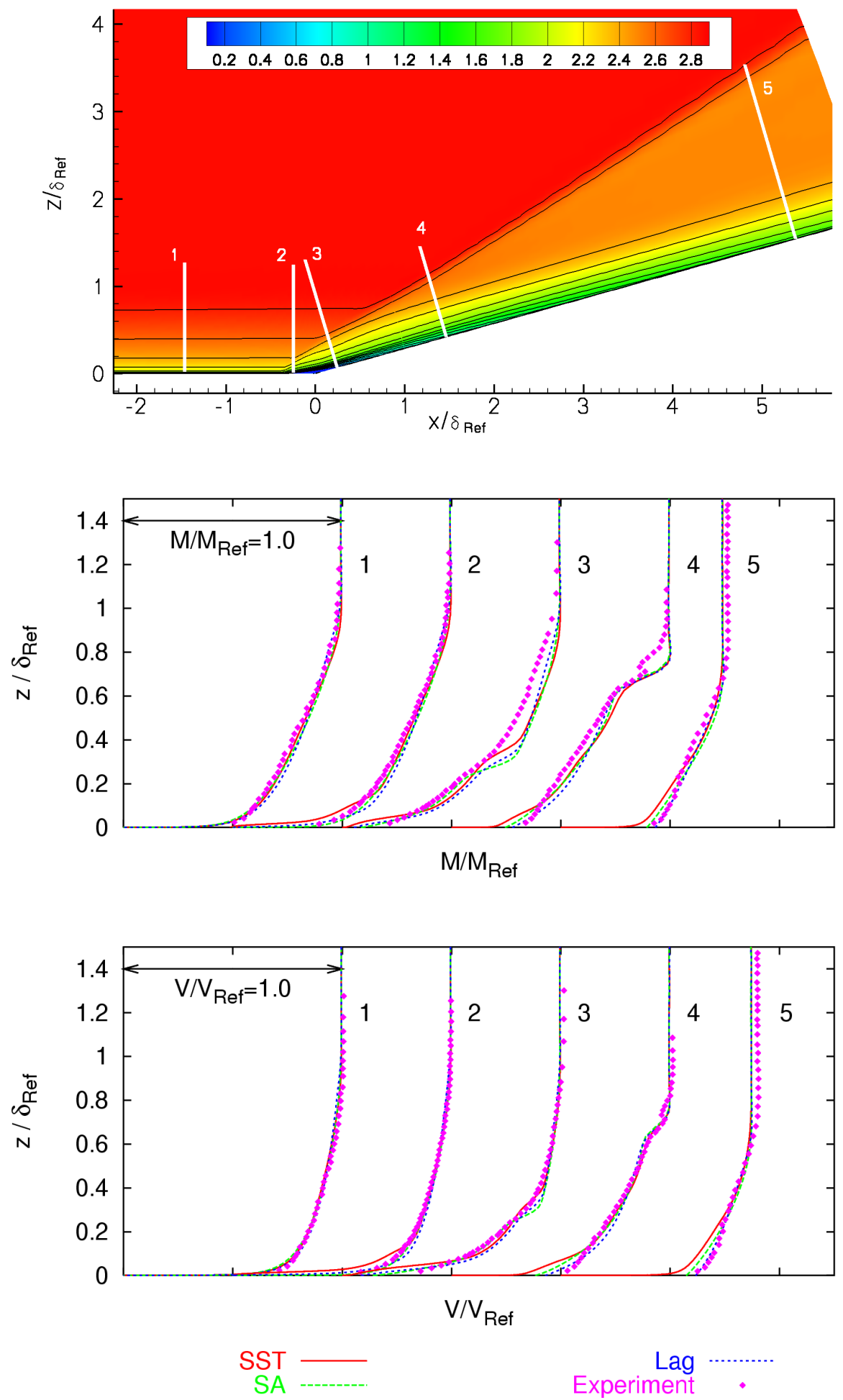

Figure 8. $16^{\circ}$ ramp: (a) Mach contours with profile locations indicated. (b) Mach profiles. (c) Velocity profiles. 

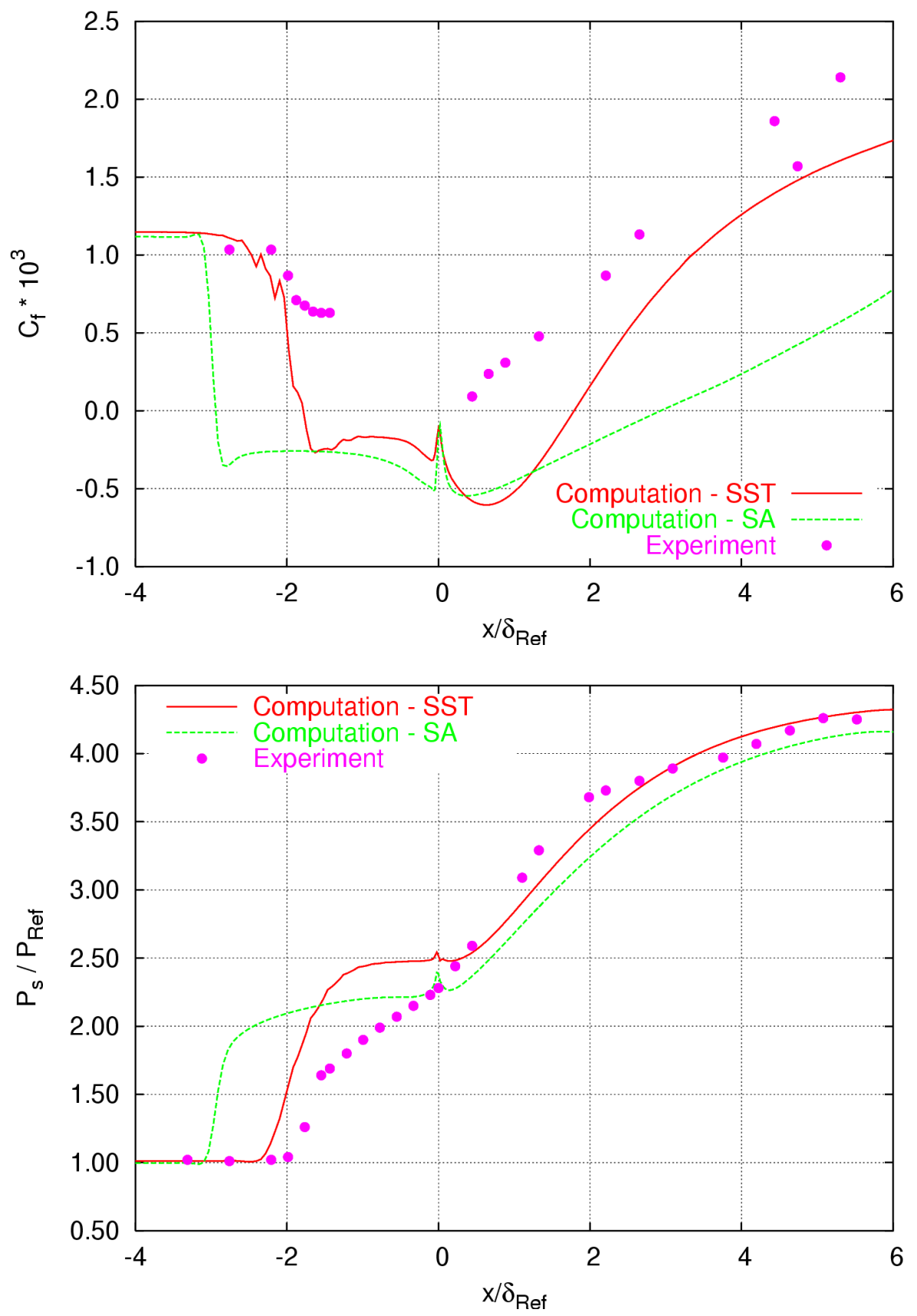

Figure 9. (a) Skin friction and (b) Surface pressures on $24^{\circ}$ ramp. Note that SA curves are from a steady-state solution and the SST curves are a time averaged unsteady solution. 


\section{Conclusions and Future Work}

The primary objective of this paper is to evaluate the capability of the OVERFLOW code to accurately predict heat transfer in turbulent hypersonic flows and identify what aspects of the code need improvement to make OVERFLOW a useful aerothermal analysis tool. Multiple flat plate and compression corner experiments have been selected for the evaluation of specific features of turbulent high-speed boundary layers. Preliminary results seem to show that for weak shock-boundary layer interactions, the SA, SST, and Lag turbulence models behave similarly, however differences begin to appear for a $16^{\circ} \mathrm{ramp}$ at Mach 3 . All of the models predict separation where the experiments reports none, thought the SST model is the worst at overpredicting the separation size. The Lag model appears to do the best overall of the three models for the few cases shown in this paper. Current and future work in this study is focused on obtaining high-quality solutions with rigorous grid resolution studies for each test case. It appears that some of the steeper ramp angles may require special treatment (i.e. running unsteady), and these methods will be explored.

\section{Acknowledgments}

This work is supported by the NASA Johnson Space Center under Grant No. NNJ04HI12G. We would also like to thank Dr. Don Rizzetta for providing us with the solution files for his LES simulations, Dr. Gatski for providing information of his DNS simulations, and Dr. Dave Kuntz and Dr. Lex Smits for providing us with documents and information of each of their experiments.

\section{References}

\footnotetext{
${ }^{1}$ Jespersen, D. C., Pulliam, T. H., and Buning, P. G., "Recent Enhancements to OVERFLOW," AIAA 35th Aerospace Sciences Meeting, Reno, NV, January 1997, AIAA 97-0644.

${ }^{2}$ Buning, P., Jespersen, D., Pulliam, T., Klopfer, G., Chan, W., Slotnick, J., Krist, S., , and Renze, K., OVERFLOW User's Manual, Version 1.8s, NASA Langley Research Center, 2000.

${ }^{3}$ Chan, W. M., Gomez, R. J., Rogers, S. E., and Buning, P. G., "Best Practices in Overset Grid Generation," 32nd AIAA Fluid Dynamics Conference, St. Louis, MO, June 2002, AIAA 2002-3191.

${ }^{4}$ Swanson, R. and Turkel, E., "On Central-Difference and Upwind Schemes," Tech. Rep. 182061, NASA Langley Research Center, Hampton, VA, June 1993, NASA Contractor Report (ICASE).

${ }^{5}$ Olsen, M. E. and Prabhu, D. K., "Application of OVERFLOW to Hypersonic Perfect Gas Flowfields," 15th AIAA Computational Fluid Dynamics Conference, Anaheim, CA, June 2001, AIAA 2001-2664.

${ }^{6}$ Lillard, R. P. and Dries, K. M., "Laminar Heating Validation of the OVERFLOW Code," AIAA 43rd Aerospace Sciences Meeting, Reno, NV, January 2005, AIAA 2005-689.

${ }^{7}$ Spalart, P. R. and Allmaras, S. R., "A One-Equation Turbulence Model for Aerodynamic Flows," AIAA 30th Aerospace Sciences Meeting, Reno, NV, January 1992, AIAA 92-0439.

${ }^{8}$ Menter, F., Grotjans, H., and Unger, F., "Numerical Aspects of Turbulence Modelling for the Reynolds Averaged NavierStokes Equations," VKI Lecture Series, March 1997, pp. 1-45.

${ }^{9}$ Olsen, M. E. and Coakley, T. J., "The Lag Model, a Turbulence Model for Non Equilibrium Flows," 15th AIAA Computational Fluid Dynamics Converence, Anaheim, CA, June 2001, AIAA 2001-2564.

${ }^{10}$ Cary, A. M., Turbulent Boundary Layer Heat Transfer and Transition Measurements With Extreme Surface Cooling in Hypersonic Flows, MS Thesis, University of Virginia, Charlottesville, VA, August 1969.

${ }^{11}$ Gatski, T. B. and Erlebacher, G., "Numerical Sumulation of a Spatially Evolving Supersonic Turbulent Boundary Layer," Tech. Rep. NASA TM 211934, NASA Langley Research Center, Hampton, VA, September 2002.

${ }^{12}$ Smits, A. and Muck, K., "Experimental Study of Three Shock Wave/Turbulent Boundary Layer Interactions," Journal of Fluid Mechanics, Vol. 182, Sept. 1987, pp. 291-314.

${ }^{13}$ Kuntz, D., Amatucci, V. A., and Addy, A. L., "Turbulent Boundary-Layer Properties Downstream of the ShockWave/Boundary-Layer Interaction," AIAA Journal, Vol. 25, May 1987, pp. 668-675.

${ }^{14}$ Kuntz, D., An Experimental Investigation of the Shock Wave-Turbulent Boundary Layer Interaction, PhD Thesis, University of Illinois, Urbana-Champaign, May 1985.

${ }^{15}$ Settles, G. S., Fitzpatrick, T. J., and Bogdonoff, S. M., "Detailed Study of Attached and Separated Compression Corner Flowfields in High Reynolds Number Supersonic Flow," AIAA Journal, Vol. 17, No. 6, June 1979, pp. 579-585.

${ }^{16}$ Settles, G. S. and Dodson, L. J., "Hypersonic Shock/Boundary-Layer Interaction Database: New and Corrected Data," Tech. Rep. 177638, Pennsylvania State University, University Park, PA, April 1994, NASA Contractor Report.

${ }^{17}$ Rizzetta, D. P., Visbal, M. R., and Gaitonde, D. V., "Large-Eddy SImulation of Supersonic Compression-Ramp Flow by High-Order Method," AIAA Journal, Vol. 39, December 2001, pp. 2283-2292.

${ }^{18}$ Rizzetta, D. P., Visbal, M. R., and Gaitonde, D. V., "Application of Large-Eddy SImulation to Supersonic Compression Ramps," AIAA Journal, Vol. 40, August 2002, pp. 1574-1581.

${ }^{19}$ Roache, P. J., "Perspective: A MEthod for Uniform Reporting of Grid Refinement Studies," Journal of Fluids Engineering, Vol. 116, No. 3, 1994, pp. 405-413.
} 